\title{
Intimate partner violence in early adolescence: The role of gender, socioeconomic factors and the school
}

\author{
A J Mason-Jones, ${ }^{1,2} \mathrm{PhD}, \mathrm{MPH}, \mathrm{MSc}, \mathrm{RGN}, \mathrm{RHV}$; P De Koker ${ }^{2,3} \mathrm{MA}$; S M Eggers, ${ }^{4} \mathrm{MSc}$; C Mathews, ${ }^{5,2} \mathrm{PhD}$; \\ M Temmerman, ${ }^{3,6} \mathrm{MB} \mathrm{ChB}, \mathrm{PhD}$; E Leye, ${ }^{3} \mathrm{PhD} ; \mathbf{P}$ J de Vries, ${ }^{2} \mathrm{MB} \mathrm{ChB}, \mathrm{MRCPsych}, \mathrm{PhD} ; \mathbf{H}$ de Vries, ${ }^{4} \mathrm{PhD}$ \\ ${ }^{1}$ Department of Health Sciences, University of York, UK \\ ${ }^{2}$ Adolescent Health Research Unit, Division of Child and Adolescent Psychiatry, Department of Psychiatry and Mental Health, \\ Faculty of Health Sciences, University of Cape Town, South Africa \\ ${ }^{3}$ International Centre for Reproductive Health, Faculty of Medicine and Health Sciences, Ghent University, Belgium \\ ${ }^{4}$ Department of Health Promotion, School for Public Health and Primary Care (CAPHRI), Maastricht University, Netherlands \\ ${ }^{5}$ Health Systems Research Unit, South African Medical Research Council, Cape Town, South Africa \\ ${ }^{6}$ Center of Excellence for Women and Child Health, Aga Khan University, Nairobi, Kenya
}

Corresponding author: A J Mason-Jones (amanda.mason-jones@york.ac.uk)

\begin{abstract}
Background. Intimate partner violence (IPV) among adolescents is common worldwide, but our understanding of perpetration, gender differences and the role of social-ecological factors remains limited.

Objectives. To explore the prevalence of physical and sexual IPV perpetration and victimisation by gender, and associated risk and protective factors.

Methods. Young adolescents $(N=2839)$ from 41 randomly selected public high schools in the Western Cape region of South Africa (SA), participating in the PREPARE study, completed a self-administered questionnaire.

Results. The participants' mean age was 13.65 years (standard deviation 1.01), with 19.1\% (541/2 839) reporting being victims/survivors of IPV and 13.0\% (370/2 839) reporting perpetrating IPV. Girls were less likely to report being a victim/survivor of physical IPV (odds ratio (OR) 0.72 ; $95 \%$ confidence interval (CI) 0.57 - 0.92) and less likely to be a perpetrator of sexual IPV than boys (OR 0.33 ; $95 \%$ CI $0.21-0.52$ ). Factors associated with perpetration of physical and sexual IPV were similar and included being a victim/survivor (physical IPV: OR 12.42; 95\% CI 8.89 - 17.36, sexual IPV: OR 20.76; 95\% CI 11.67 - 36.93), being older (physical IPV: OR 1.26; 95\% CI 1.08 - 1.47, sexual IPV: OR 1.36; 95\% CI 1.14 - 1.62 ), having lower scores on school connectedness (physical IPV: OR 0.59; 95\% CI 0.46 - 0.75, sexual IPV: OR 0.56; $95 \%$ CI 0.42 - 0.76) and scoring lower on feelings of school safety (physical IPV: OR 0.66; 95\% CI 0.57 - 0.77, sexual IPV: OR 0.50; $95 \%$ CI $0.40-0.62$ ).

Conclusions. Physical and sexual IPV was commonly reported among young adolescents in SA. Further qualitative exploration of the role of reciprocal violence by gender is needed, and the role of 'school climate'-related factors should be taken into account when developing preventive interventions.
\end{abstract}

S Afr Med J 2016;106(5):502-509. DOI:10.7196/SAMJ.2016.v106i5.9770

Intimate partner violence (IPV) is defined as 'any behaviour within an intimate relationship that causes physical, psychological or sexual harm to those in the relationship. ${ }^{[1]}$ It can include humiliation, intimidation and controlling behaviour such as monitoring movements and restricting access to resources or healthcare and physical and sexual violence such as slapping, beating, forced sex or other forms of coercion, which can result in severe injury and death. ${ }^{[2]}$ Those experiencing IPV may present to healthcare services with physical injury, ${ }^{[3]}$ depression, or suicidal ideation and suicide attempts. ${ }^{[4]}$ Studies worldwide have shown that physical, psychological and sexual violence vary widely across countries and have been reported in $10-50 \%$ of relationships for individuals aged up to 19 years, including in the USA, India, Nigeria, China, ${ }^{[5]}$ the UK, ${ }^{[6,7]}$ Tanzania $^{[8]}$ and South Africa (SA). ${ }^{[5,8,9]}$ Most studies have reported that psychological violence is most common, followed by physical violence and then sexual violence. ${ }^{[10,11]}$ Risk factors for perpetration and victimisation of IPV in adolescent relationships also vary between countries, and associations with higher age, ${ }^{[12]}$ not being raised by a biological mother, ${ }^{[11]}$ higher maternal education, ${ }^{[8]}$ substance use, particularly alcohol, ${ }^{[9,11,13]}$ previous maltreatment, violence in the home and aggressive peer networks, ${ }^{[10]}$ especially at school, ${ }^{[13]}$ and attitudes supportive of male superiority, ${ }^{[6,9]}$ and for girls having an older partner, ${ }^{[8,10]}$ have been reported. Protective factors include religion, ${ }^{[8]}$ holding prosocial beliefs, ${ }^{[13]}$ and parental monitoring for boys, and for girls with no family conflict. ${ }^{[13]}$ The evidence is equivocal for disadvantaged background ${ }^{[6-8,11,12]}$ and gender, especially in this very young age group, with some studies saying that experiencing violence may be more common for males ${ }^{[8]}$ and others for females. ${ }^{[6,9,12]}$ The most severe consequences of IPV nevertheless show a clear gender difference, worldwide ${ }^{[1,2]}$ and particularly in SA, which has the highest reported rate of intimate femicide in the world. ${ }^{[14]}$

Although several studies have examined IPV among adolescents in SA, gender differences in perpetration and victimisation for young adolescents required further exploration. More evidence was also needed for the factors that may be associated with IPV perpetration and victimisation, including socioeconomic status (SES), family-related factors and the potential role of the school environment.

\section{Objectives}

To: (i) explore the prevalence of physical and sexual IPV perpetration and victimisation by gender in a representative sample of adolescents who were part of a school-based study; (ii) determine whether 
there was an association between perpetration and victimisation for physical and sexual IPV; and (iii) examine the risk and protective factors for physical and sexual IPV perpetration and victimisation, and whether these factors differed by gender.

\section{Methods}

Baseline data were collected for the PREPARE study ('Promoting sexual and reproductive health among adolescents in southern and eastern Africa - mobilising schools, parents and communities'), a cluster randomised controlled trial conducted in 41 public high schools in the Western Cape Province of SA to evaluate an HIV prevention programme that focused on IPV and sexual violence reduction (PREPARE project: ISRCTN56270821). The PREPARE study was approved by the Western Norway Regional Committee for Medical and Health Research Ethics, the Human Research Ethics Committee at the University of Cape Town, SA, and the Western Cape Provincial Department of Education. As some of the questions were of a sensitive nature, appropriate services were made available to participants. A total of 6244 grade 8 students (average age 13 years) were invited to participate, of whom 55.3\% (3 451) returned a signed parental/legal guardian consent form and also signed an assent form. A self-administered paper questionnaire in three languages (English, Xhosa and Afrikaans) resembling a young persons' magazine was administered in February and March 2013. After listwise deletion and deleting of records of students who did not report on gender or had missing data on IPV measures, the final sample for the purposes of this study was 2839 .

\section{Measurements}

Multiple-choice questions covered sociodemographic information and indicators hypothesised to be risk and protective factors for perpetration and victimisation of violence in intimate relationships.

\section{Sociodemographic factors}

These included whether participants identified themselves as 'white', 'black', 'coloured' or 'other' ('race' classifications previously imposed by the apartheid government that continue to be associated with health inequalities and inequities in healthcare provision), age, gender and orphan status (maternal/paternal orphan: 'Is your mother/father alive?' ( $0=$ no, $1=$ yes, $2=\mathrm{I}$ don't know; double orphan: mother and father have died $(0=$ no, $1=$ yes). SES was assessed by using a family affluence scale. Scores were calculated by adding up the individual scores $(0=$ no, $1=$ yes $)$ for each indicator of socioeconomic wellbeing (e.g. having tap water inside the house, electricity, telephone). This resulted in an SES score ranging from 0 (having none of the items) to 8 (having all of the items).

\section{Intimate partner violence}

Items measuring IPV were adapted from the World Health Organization multicountry study. ${ }^{[1]}$ Variables associated with physical and sexual IPV perpetrator/victim status within the past 6 months were assessed with a 'yes' answer (score of 1 ) to each question scored as the participant being a perpetrator or a victim of physical and sexual IPV, respectively.

Perpetration of physical IPV was assessed by asking how often the participant had hit, pushed, kicked, choked or burned their boyfriend/girlfriend ( $0=$ never, $1=$ at least once).

Victimisation of physical IPV was assessed by asking how often the participant had been hit, pushed, kicked, choked or burned by a boyfriend/girlfriend ( $0=$ never, $1=$ at least once).

Perpetration of sexual IPV was assessed by asking how often the participant had forced their boyfriend/girlfriend to have sex $(0=$ never, $1=$ at least once).
Victimisation of sexual IPV was assessed by asking how often the participant had been forced to have sex by a boyfriend/girlfriend $(0=$ never, $1=$ at least once).

\section{School-related factors}

The following measures were also incorporated in the questionnaire.

School performance was assessed by asking participants 'Have you ever repeated a school year?' ( $0=$ no, $1=$ yes $)$, and 'Last year, how well did you do in school compared with the others in your class?' (answer options: 'I was among the best of my class' (representing a high score), 'I was better than average, 'I was about average,' 'I was below average' and 'I was among the worst of my class' (representing a low score)). A higher score meant higher/better school performance.

School climate questions from the Yale School of Medicine High School Student Climate Survey were included. A five-point Likert scale was used (strongly agree, agree, not sure, disagree and strongly disagree), which were then dichotomised to ' 0 for no' and ' 1 for yes'. For the purposes of the analysis, we computed the mean score of these questions and calculated the Cronbach's alpha, which was $0.67,0.82$, and 0.78 for school safety, connectedness and appearance, respectively. A higher score meant safer/more connected or better school climate.

School safety was assessed by asking participants to agree/disagree with statements such as 'Some students at my school often say that they hit or beat others', 'At my school, it is easy for criminals to come into the school grounds' and 'Students often get hurt at my school.'

School connectedness was assessed by asking participants to agree/ disagree with statements such as 'I like school', 'I look forward to going to school,' 'I try hard at school' and 'Finishing high school is important to me'.

School appearance was assessed by asking participants to agree/ disagree with statements such as 'My school building is clean', 'I like the way my school looks' and 'My school is well maintained'.

\section{Analysis}

Data were analysed using SPSS 22.0 and STATA 13.0. Sample characteristics were described with $\chi^{2}$ tests and analysis of variance (ANOVA), with means and standard deviations (SDs) calculated for continuous variables. To determine whether there was an association between perpetration and victimisation for physical and sexual IPV and to explore the association of specific risk and protective factors of interest, multiple logistic and linear regression models were applied. Data were stratified by gender and all analyses were adjusted for the clustered design (students nested within schools), by using the mixed-models generalised estimation equations in STATA to avoid underestimation of the standard errors. Those who reported being perpetrators of IPV were compared with non-perpetrators of IPV, and survivors of IPV were compared with those who did not report experiencing IPV.

\section{Results}

The mean age of the participants was 13.65 years (SD 1.01), 60.9\% ( $1729 / 2$ 839) were girls, and $57.6 \%(n=1629)$ self-identified as 'coloured', $34.6 \%(n=977)$ as 'black', $5.0 \%(n=141)$ as 'white' and $2.8 \%$ $(n=80)$ as 'other'. More than 1 in $5(21.8 \%, n=604)$ had repeated a school year. Thirteen percent (370/2 839) of participants reported perpetrating IPV and 19.1\% (541/2 839) reported being victims/ survivors of IPV.

\section{Prevalence rates of physical and sexual IPV according to gender}

Ten percent of our sample overall (284/2 839) reported being perpetrators of physical IPV and $15.8 \%$ (449/2 839) were victims/ 
survivors of physical IPV. Of the boys, $13.8 \%(153 / 1110)$ reported physical IPV perpetration and 21.1\% (234/1 110) physical IPV victimisation. Of the girls, $7.6 \%$ (131/1 729) reported physical IPV perpetration and $12.4 \%(215 / 1729)$ physical IPV victimisation. Overall, $5.9 \%$ (168/2 839) of participants, reported being perpetrators of sexual IPV and $7.3 \%(206 / 2$ 839) being victims. For sexual IPV, prevalence rates for boys were $10.8 \%$ (120/1 110) for perpetration and $11.1 \%$ (123/1 110) for victimisation. For girls the rates were $2.8 \%(48 / 1729)$ for sexual IPV perpetration and $4.8 \%$ (83/1 729) for sexual IPV victimisation. Boys reported significantly more perpetration as well as more victimisation than girls for physical IPV (Table 1) and sexual IPV (Table 2).

Compared with male non-perpetrators of physical IPV, male perpetrators were older (14.31 (SD 1.09) v. 13.73 (SD 1.10) years), were more likely to identify as 'white' $(10.3 \%(15 / 153) \mathrm{v}$. $5.3 \%(50 / 957))$ or 'black' (51\% (74/153) v. $34.1 \%(321 / 957))$, were more likely to have lost their father due to death $(16.7 \%$ (24/153) v. 10.9\% (101/957)), had lower mean SES scores (5.70 (SD 1.65) v. 6.16 (SD 1.53)), were more likely to have repeated a school year $(47.8 \%(65 / 153)$ v. $25.2 \%(228 / 957)$ ), and had lower mean scores for school performance (3.70 (SD 0.98) v. 3.91 (SD 0.88)), school connectedness (4.19 (SD 0.86) v. 4.53 (SD 0.72)), and feelings of school safety (2.44 (SD 0.98) v. 2.94 (SD 1.04)) (Table 1). A significant difference in ethnicity was found when comparing male physical IPV perpetrators with non-perpetrators, with those who identified as 'coloured' being less likely to be perpetrators of IPV (38.6\% $(56 / 153)$ v. $57.5 \%(542 / 957))$. Similar results were reported for male survivors of IPV v. males who did not report experiencing IPV, although paternal orphanhood and school performance were no longer statistically significant. In addition, male survivors of IPV scored lower on perceptions of school appearance.

Compared with female non-perpetrators of physical IPV, perpetrators were older (13.74 (SD 0.76) v. 13.51 (SD 0.90) years), were more likely to have lost their father due to death $(20.5 \%$ $(26 / 131)$ v. $10.4 \%(160 / 131))$, and had lower scores for school connectedness (4.46 (SD 0.76) v. 4.64 (SD 0.57)), lower

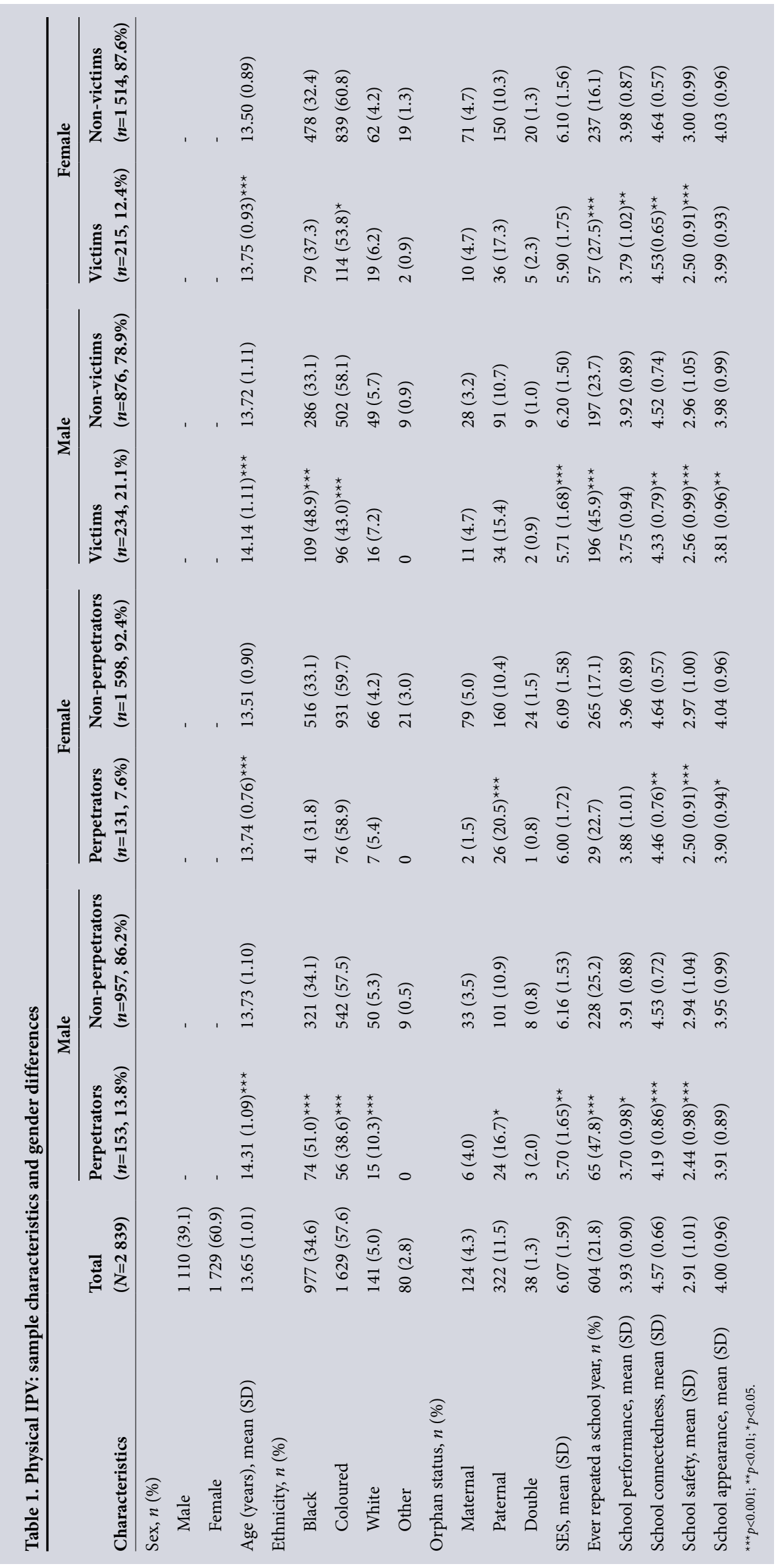




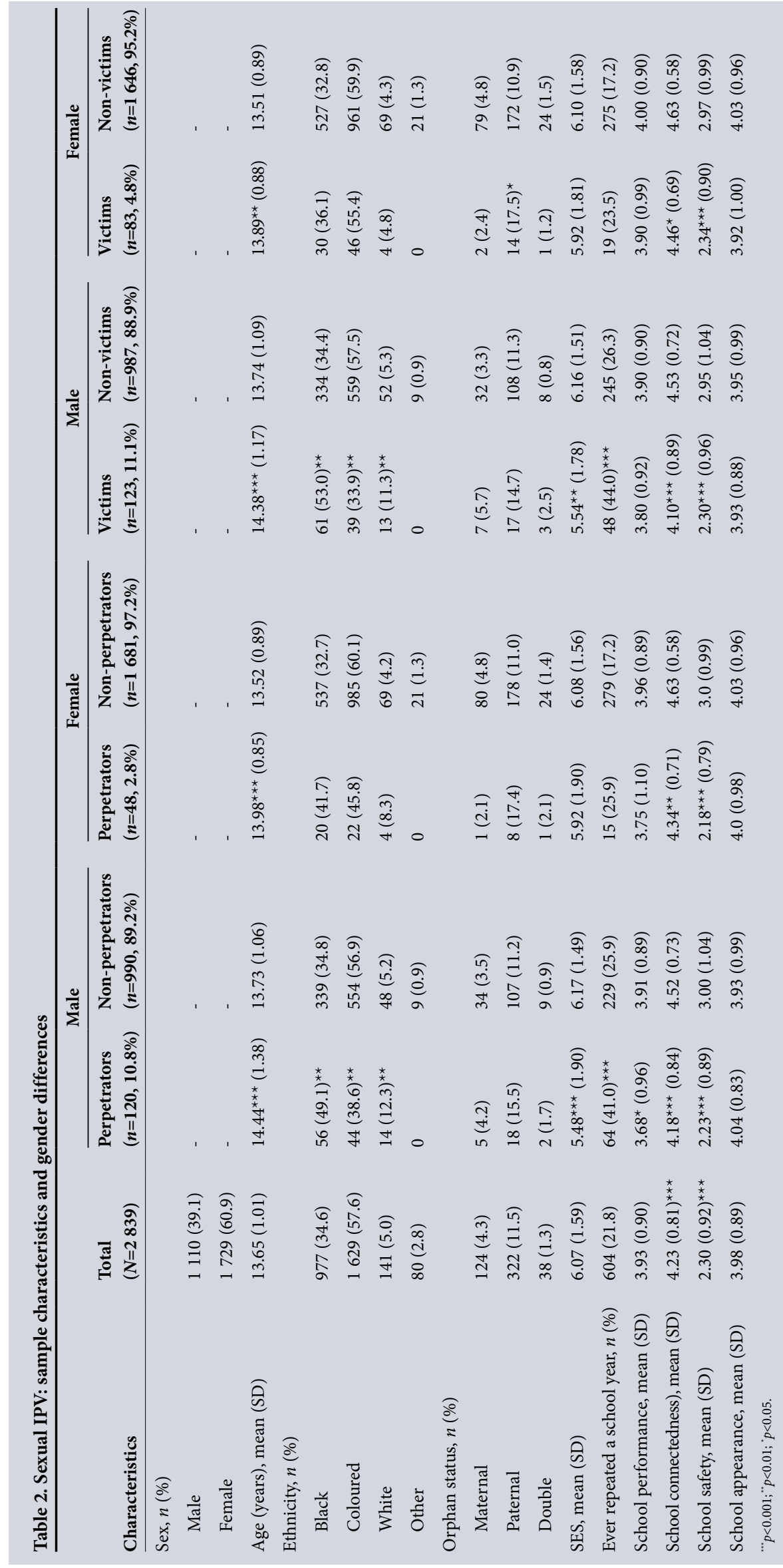

feelings of school safety (2.50 (SD 0.91) v. 2.97 (SD 1.00)) and less favourable views about their school's appearance (3.90 (SD 0.94) v. 4.04 (SD (0.96)) (Table 1). Similar results were found for female survivors of IPV compared with their counterparts who did not report experiencing IPV, except that they were statistically significantly more likely to have repeated a school year $(27.5 \%$ $(57 / 215)$ v. $16.1 \%(237 / 1514))$ and to have a lower mean score for school performance (3.79 (SD 1.02) v. 3.98 (SD 0.87)), and less likely to identify as 'coloured' $(53.8 \%(114 / 215)$ v. $60.8 \%$ (839/1 514)).

Sexual IPV followed a similar pattern to physical IPV (Table 2). Both male and female perpetrators and survivors of IPV were older, were more likely to identify as 'black' or 'white' and less likely to identify as 'coloured', and had lower school connectedness and feelings of school safety compared with their male and female counterparts who were neither perpetrators nor survivors of IPV. In addition, female survivors of IPV were more likely to have a father who had died $(17.5 \%(14 / 83) \mathrm{v}$. $10.9 \%(172 / 1646))$, male perpetrators and survivors had lower SES (5.48 (SD 1.90) v. 6.17 (SD 1.49) for perpetrators and 5.54 (SD 1.78) v. 6.16 (SD 1.51) for survivors) and were more likely to have repeated a school year $(41.0 \%(64 / 120)$ v. $25.9 \%(229 / 990)$ for perpetrators and $44.0 \%(48 / 123)$ v. 26.3 (245/987) for survivors), and male perpetrators were also more likely to have poor scores for school performance (3.68 (SD 0.96) v. 3.91 (SD 0.89)).

\section{Associations between perpetration and victimisation for physical and sexual IPV}

Bivariate correlations were found for boys and girls regarding perpetration and victimisation of both physical and sexual IPV (Table 3). Multiple linear and logistic regression models showed that factors associated with perpetration of physical IPV for the whole sample were being a victim of physical IPV (odds ratio (OR) 12.42; 95\% confidence interval (CI) 8.89 - 17.36), being older (OR 1.26; 95\% CI 1.08 - 1.47), having lower school connectedness (OR 0.59; 95\% CI 0.46 - 0.75) and scoring lower on feelings of school safety (OR 0.66 ; 95\% CI 0.57 - 0.77) (Table 4). For boys, 


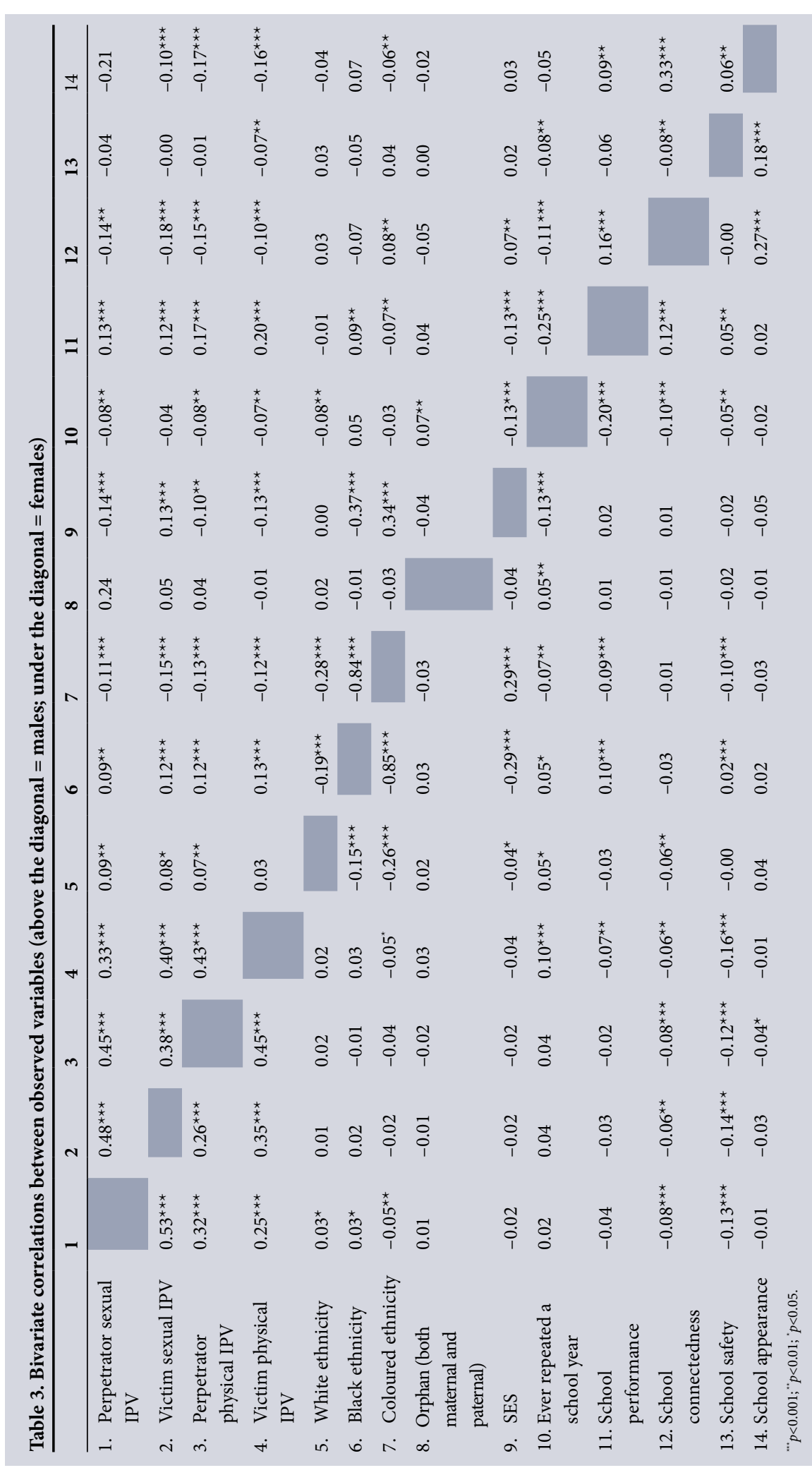

factors associated with being a perpetrator of physical IPV were being a victim of physical IPV (OR 5.75; 95\% CI 3.65 - 9.08), being older (OR 1.23; 95\% CI 1.04 - 1.47), lower school connectedness (OR 0.53; 95\% CI $0.39-0.71)$, lower feelings of school safety (OR 0.61; 95\% CI $0.50-0.74$ ) and a negative view of their school's appearance (OR 1.29; 95\% CI 1.01 - 1.62). For girls, the factors associated with physical IPV perpetration were being a victim of physical IPV themselves (OR 17.69; 95\% CI 10.95 - 28.57) and having lower school connectedness (OR 0.66; 95\% CI 0.58 - 0.94) and feelings of school safety (OR 0.74; 95\% CI 0.58 - 0.94).

For the whole sample, girls were less likely to be a victim/survivor of physical IPV than boys (OR 0.72; 95\% CI 0.57 0.92), while being a perpetrator of physical IPV (OR 12.38; 95\% CI 8.80 17.43), having repeated a school year (OR 1.72; 95\% CI $1.24-2.38$ ) and a lower mean score on feelings of school safety (OR 0.72; 95\% CI 0.63 - 0.83) were associated with higher odds of being a victim/survivor of physical IPV (Table 4). For boys, factors associated with being a victim/survivor of physical IPV were being a perpetrator of physical IPV (OR 9.07; 95\% CI 5.58 14.74), having repeated a school year (OR 2.08; 95\% CI 1.34 - 3.25) and reporting lower feelings of school safety (OR 0.79; 95\% CI 0.68 - 0.91). For girls, factors associated with being a victim/ survivor of physical IPV were being a perpetrator of physical IPV (OR 17.65; 95\% CI 10.87 - 28.66) and reporting lower feelings of school safety (OR 0.64 ; 95\% CI 0.54 - 0.79).

Girls were less likely to perpetrate sexual IPV than boys (OR 0.33; 95\% CI 0.21 0.52 ), and for the whole sample, being a victim of sexual IPV (OR 20.76; 95\% CI 11.67 - 36.93), being older (OR 1.36; 95\% CI 1.14 - 1.62), having lower school connectedness (OR 0.56; 95\% CI 0.42 0.76) and reporting lower feelings of school safety (OR 0.50; 95\% CI 0.40 - 0.62) were all associated with perpetration of sexual IPV. These associations were similar for both boys and girls who perpetrated sexual IPV. Both groups had higher odds of having been victims of sexual IPV (OR 11.65; 95\% CI 5.72 - 23.72 for boys and OR 53.72; 95\% CI 20.23 - 142.65 for girls). Boys who perpetrated sexual IPV also reported lower scores on school appearance (OR 1.34; 95\% CI 1.08 - 1.68) (Table 5). For the whole sample, the only factor associated with being a victim/survivor of sexual IPV was being a perpetrator (OR 20.39; 95\% CI $11.39-36.51)$. The odds varied for boys (OR 11.65; 95\% CI 5.72 - 23.75) and girls (OR 53.16; 95\% CI 19.20 147.17), with boys also scoring lower on school connectedness (OR 0.54; 95\% CI $0.36-0.81)$ and feelings of school safety (OR 0.57; 95\% CI 0.40 - 0.80), and girls for school safety only (OR 0.61; 95\% CI 0.45 0.82 ).

\section{Discussion}

Our study aimed to examine perpetration and victimisation of intimate partner 


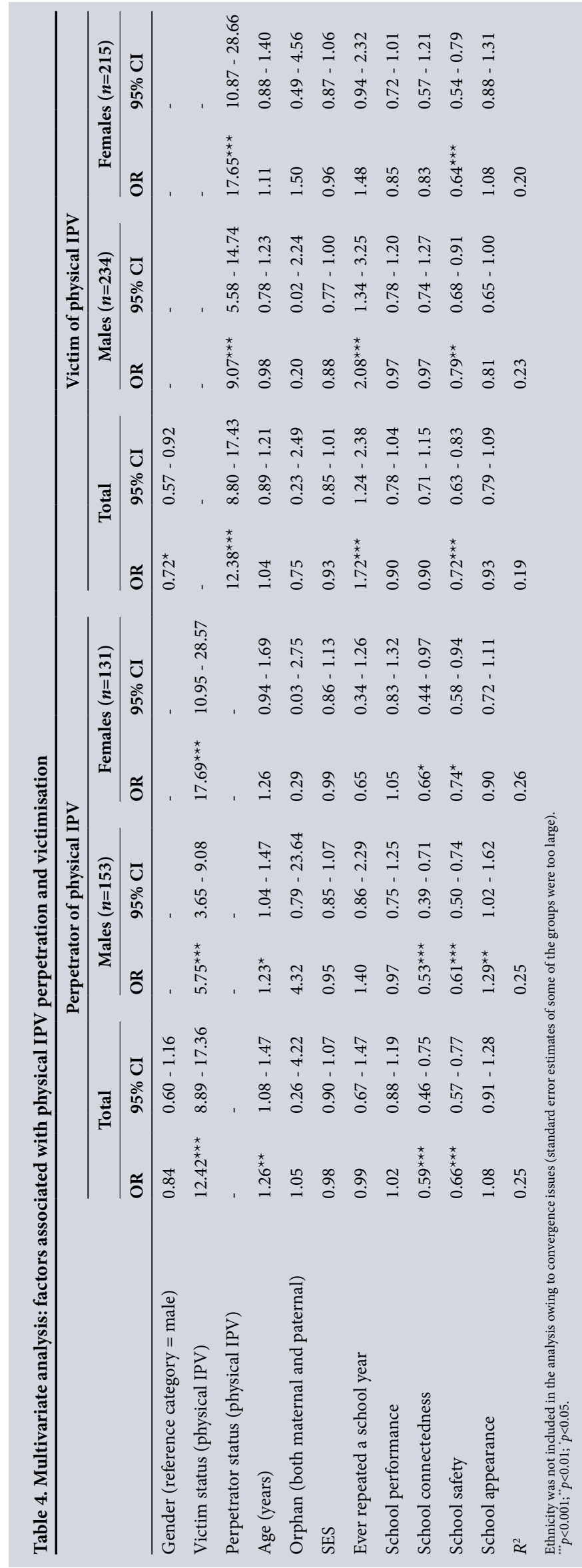

violence in a sample of young schoolgoing adolescents and to explore associations with gender, socioeconomic variables and school factors. Ten percent of participants reported perpetrating physical violence and $5.9 \%$ sexual violence in their intimate relationships in the previous 6 months, while $21.1 \%$ of boys and $12.4 \%$ of girls reported being victims of physical IPV and $11.1 \%$ of boys and $4.8 \%$ of girls being victims of sexual IPV. This is alarming considering that the mean age of the participants was only 13 years and reporting was only for the previous 6 months. Male perpetration of physical and sexual IPV was associated with being a victim of physical/sexual IPV, higher age, low scores on school connectedness and feelings of school safety, and more negative feelings about school appearance. Being a male victim/survivor of physical IPV was associated with being a perpetrator, having repeated a school year and lower scores on feelings of school safety, while for males sexual IPV victimisation was associated with being a perpetrator and having lower scores for school connectedness and feelings of school safety. Female perpetration of physical and sexual IPV was associated with being a victim of physical/sexual IPV and lower scores on school connectedness and feelings of school safety plus higher age for sexual IPV only. Being a female victim/survivor of both physical and sexual IPV was associated with being a perpetrator and lower scores on feelings of school safety.

The prevalences of physical and sexual IPV perpetration and victimisation were higher among boys than among girls in our study, which is similar to rates reported for similar populations in $\mathrm{SA}^{[8]}$ and for similar age groups in the USA, ${ }^{[4,13]}$ but different to evidence from other SA studies, one of which asked about IPV victimisation in the previous 3 month $s^{[9]}$ and one that asked about physical IPV only. ${ }^{[12]}$ The victimisation prevalence for boys seems counterintuitive considering the high fatalities resulting from IPV for adult women in SA, ${ }^{[14]}$ and its impact on health and wellbeing for women worldwide. ${ }^{[1-3]}$ The results could be due to differential reporting between boys and girls, differences in context of the violence, ${ }^{[6]}$ and perhaps that boys are more likely to be pressured into having relationships at a younger age than girls, which may increase their exposure to IPV. However, it is not clear from our data with whom the boys were having relationships. There have been anecdotal reports of younger boys engaging in relationships with much older adult women in SA, so research is needed to explore this in more depth. We also do not have details about the severity of the violence, and it could be that as males get older perpetration of violence against females becomes more extreme, is more likely to inflict injury ${ }^{[15]}$ and is more likely to result in fatalities. ${ }^{[1,2,14]}$ The finding that those who identified as 'coloured' were less likely to engage in IPV at this age also needs further examination, as there may be protective factors related to religion, prosocial beliefs or parental monitoring ${ }^{[8,13]}$ in this group.

We also found very strong associations between perpetration and victimisation for physical and sexual IPV. Victimisation was the strongest predictor for perpetration, and vice versa. Both boys and girls who were perpetrators of physical IPV had increased odds for being victims of physical IPV, although the odds for girls were much higher compared with boys, with more extreme differences for sexual IPV for girls compared with boys, although the estimates were less precise. Given that this was a crosssectional study, we cannot deduce temporal associations between perpetration and victimisation, although other studies have found similar associations. ${ }^{[8,15]}$ Future investigations will benefit from exploring underlying factors in reciprocal violence using more 


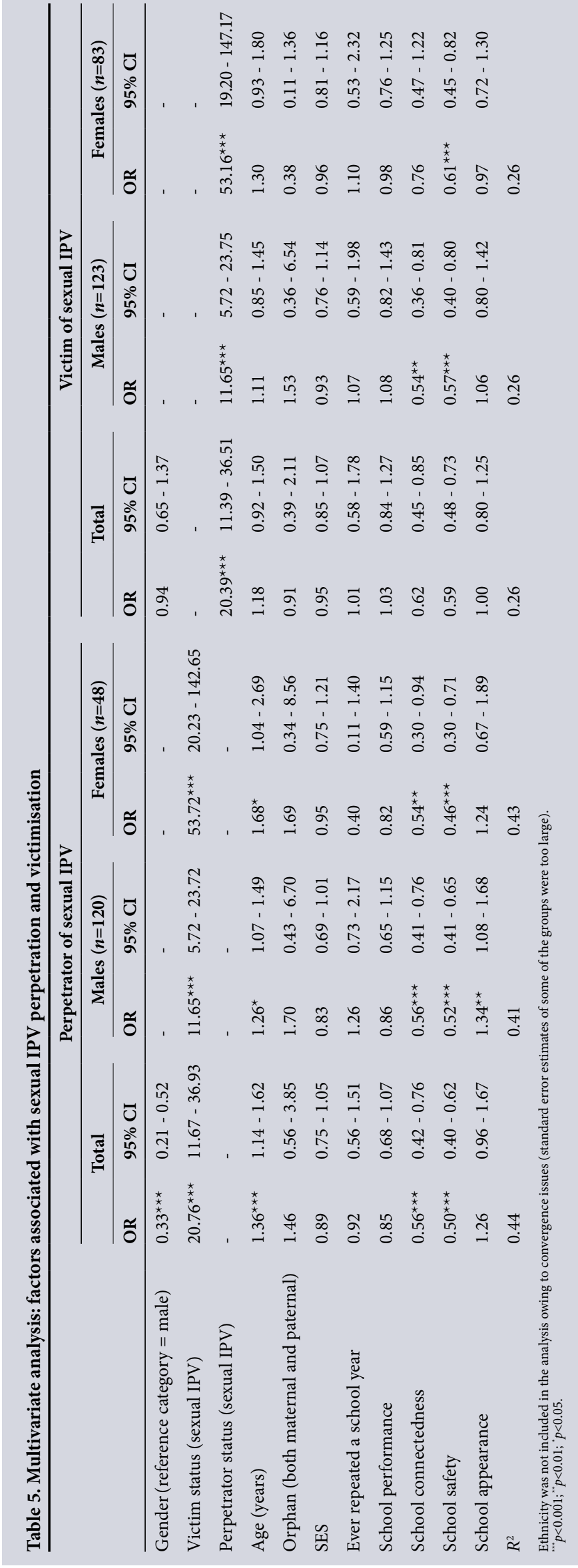

qualitative longitudinal methods to understand the context and meaning of the violence, which may provide important clues for improving interventions and recognising IPV risk both in clinical practice and in schools.

Our results also demonstrated that low scores on school connectedness and feelings of school safety were associated with physical and sexual IPV perpetration and victimisation for both boys and girls, although school performance per se was not associated with IPV. As school is an important setting for IPV prevention, the significance of school factors as potential mediators or modifiers for adolescents being or becoming perpetrators or victims of physical or sexual IPV needs to be considered. In particular, it is important to prevent dropout from school, which is known to have adverse consequences. Future research should therefore explore the role of schools and specifically school climate in preventing IPV among adolescents.

\section{Study limitations}

This study was subject to some limitations. Firstly, adolescent physical and sexual IPV is a sensitive topic for very young people and we relied on self-reports, so even though anonymity was assured, the validity of the answers could be questioned. Participants may not have defined their experiences as 'IPV', particularly the girls, ${ }^{[6]}$ and could have misunderstood questions, declined to answer, or deliberately masked perpetration or victimisation. Nevertheless, the prevalence of IPV in our study does correspond with that from studies with similar populations. ${ }^{[8,11-13]}$ Secondly, our study did not explore the characteristics of perpetrating partners who may be older or younger, or of the same or different sex. Again this is important for the development of interventions that could assist young people to report the abuse, as previous studies have shown that help-seeking by young people engaged in IPV is virtually non-existent and over half seek help from friends only. ${ }^{[10]}$ Thirdly, owing to our large sample, some statistically significant findings may not necessarily have clear predictive value for individuals.

\section{Conclusions}

Despite the potential limitations, our study presents a clear exploration of both perpetration and victimisation of physical and sexual IPV in a young adolescent population and adds new socioecological insights to the existing literature. Our findings have underlined the very high prevalence of reciprocal IPV among young boys and girls in SA and the urgent need to investigate this issue further in order to develop appropriate interventions to prevent long-term adverse health impacts. ${ }^{[-3,3,1,14,15]}$ The evidence linking demographic factors associated with IPV, including lower SES, the death of a father, and school factors such as repeating a school year and lower scores on school climate-related measures, suggests the need for proportionate universal multilevel models of intervention. Finally, more attention should be given to addressing community-level factors that can potentially protect young people from physical and sexual IPV perpetration and victimisation.

Authorship and acknowledgements. All authors fulfil the requirements for authorship, contributed to drafting the manuscript, agreed on the final version and have no conflicts of interest to declare. The project from which the data were taken is PREPARE, the full title of which is 'Promoting sexual and reproductive health among adolescents in southern and eastern Africa - mobilising schools, parents and communities. The PREPARE study was funded by the EC Health research programme (under the 7th Framework Programme), 
grant agreement number 241945. The partners and principal investigators include the University of Cape Town (Cathy Mathews), Muhimbili University of Health and Allied Sciences (Sylvia Kaaya), the University of Limpopo (Hans Onya), Makerere University (Anne Katahoire), Maastricht University (Hein de Vries), the University of Exeter (Charles Abraham), the University of Oslo (Knut-Inge Klepp) and the University of Bergen (Leif Edvard Aarø - co-ordinator). We express our gratitude to the members of the PREPARE Scientific Advisory Committee: Nancy Darling, Oberlin College, Ohio, USA; Jane Ferguson, World Health Organization, Geneva, Switzerland; Eleanor Maticka-Tyndale, University of Windsor, Ontario, Canada; and David Ross, London School of Hygiene and Tropical Medicine, UK. We are indebted to the school staff and young people for their participation in this study. See also the project homepage (http://prepare.b.uib.no/). The trial registry site and number are http:// www.controlled-trials.com/ISRCTN56270821 and ISRCTN56270821.

\section{References}

1. García-Moreno C, Jansen HA, Ellsberg M, et al. WHO Multi-country Study on Women's Health and Domestic Violence against Women: Initial Results on Prevalence, Health Outcomes and Women's Responses. Geneva: WHO Press, 2005.

2. García-Moreno C, Pallitto C, Devries K, et al. Global and Regional Estimates of Violence against Women: Prevalence and Health Effects of Intimate Partner Violence and Non-partner Sexual Violence. Women: Prevalence and $\mathrm{H}$

3. Ellsberg M, Jansen HAFM, Heise L, et al. Intimate partner violence and women's physical and mental health in the WHO Multi-country Study on Women's Health and Domestic Violence: An observational study. Lancet 2008;371(9619):1165-1172. DOI:10.1016/S0140-6736(08)60522-X
4. Nahapetyan L, Orpinas P, Song X, et al. Longitudinal association of suicidal ideation and physical dating violence among high school students. J Youth Adolesc 2014;43(4):629-640. DOI:10.1007/ s10964-013-0006-6

5. Halpern CT, Oslak SG, Young ML, et al. Partner violence among adolescents in opposite-sex romantic Halpern CT, Oslak SG, Young ML, et al. Partner violence among adolescents in opposite-sex romantic
relationships: Findings from the National Longitudinal Study of Adolescent Health. Am J Public relationships: Findings from the National Longitudinal Study of
Health 2001;91(10):1679-1685. DOI:10.2105/AJPH.91.10.1679

6. Hird MJ. An empirical study of adolescent dating aggression in the U.K. J Adolesc 2000;23(1):69-78. DOI:10.1006/jado.1999.0292

7. Wood M, Barter C, Berridge D. Standing On My Own Two Feet: Disadvantaged Teenagers, Intimate Partner Violence and Coercive Control. NSPCC, 2011. https://www.nspcc.org.uk/globalassets/ documents/research-reports/standing-own-two-feet-report.pdf (accessed 31 March 2016).

8. Wubs AG, Aarø LE, Flisher AJ, et al. Dating violence among school students in Tanzania and South Africa: Prevalence and socio-demographic variations. Scand J Public Health 2009;37(2 suppl):75-86. DOI:10.1177/1403494808091343

9. Russell M, Cupp PK, Jewkes RK, et al. Intimate partner violence among adolescents in Cape Town, South Africa. Prev Sci 2014;15(3):283-295. DOI:10.1007/s11121-013-0405-7

10. Barter C, McCarry M, Berridge D, et al. Partner Exploitation and Violence in Teenage Intimate Relationships. NSPCC, 2009. https://www.nspcc.org.uk/globalassets/documents/research-reports/ Relationships. NSPCC, 2009. https://WwW.nspcc.org.uk/globalassets/documents/research-reports/
partner-exploitation-violence-teenage-intimate-relationships-summary.pdf (accessed 31 March 2016).

11. Decker MR, Peitzmeier S, Olumide A, et al. Prevalence and health impact of intimate partner violence and non-partner sexual violence among female adolescents aged 15-19 years in vulnerable urban environments: A multi-country study. J Adolesc Health 2014;55(6):S58-S67. DOI:10.1016/j. jadohealth.2014.08.022

12. Flisher AJ, Myer L, Mèrais A, et al. Prevalence and correlates of partner violence among South African adolescents. J Child Psychol Psychiatry 2007;48(6):619-627. DOI:10.1111/j.1469-7610.2007.01711.x

13. Foshee VA, Reyes LM, Tharp AT, et al. Shared longitudinal predictors of physical peer and dating violence. J Adolesc Health 2015;56(1):106-112. DOI:10.1016/j.jadohealth.2014.08.003

14. Abrahams N, Jewkes R, Martin LJ, et al. Mortality of women from intimate partner violence in South Africa: A national epidemiological study. Violence Vict 2009;24(4):546-556. DOI:10.1891/08866708.24.4.546

15. Whitaker DJ, Haileyesus T, Swahn M, et al. Differences in frequency of violence and reported injury between relationships with reciprocal and nonreciprocal intimate partner violence. Am J Public Health 2007:97(5):941-947. DOI:10.2105/AJPH.2005.079020

Accepted 30 March 2016. 